Submitted to ApJ

Preprint typeset using $\mathrm{LT}_{\mathrm{E}} \mathrm{X}$ style emulateapj v. 6/22/04

\title{
FEEDBACK FROM CLUSTERED SOURCES DURING REIONIZATION
}

\author{
Roban Hultman Kramer, Zoltán Haiman \\ Department of Astronomy, Columbia University, 550 West 120th Street, New York, NY 100271
}

\author{
S. PENG OH \\ Department of Physics; University of California; Santa Barbara, CA 93106 \\ Submitted to ApJ
}

\begin{abstract}
The reionization history of the intergalactic medium (IGM) at high redshift $(z \gtrsim 6)$ was likely strongly shaped by several global feedback processes. Because the earliest ionizing sources formed at the locations of the rare density peaks, their spatial distribution was strongly clustered. Here we demonstrate that this clustering significantly boosts the impact of feedback processes operating at high redshift. We build a semi-analytical model to include feedback and clustering simultaneously, and apply this model to the suppression of starformation in minihalos due to photoionization. The model is built on the excursion-set-based formalism of Furlanetto, Zaldarriaga, \& Hernquist (2004), which incorporates the clustering of ionizing sources, and which we here extend to include suppression of star formation in minihalos. We find that clustering increases the mean HII bubble size by a factor of several, and it dramatically increases the fraction of minihalos that are suppressed, by a factor of up to $\sim 60$ relative to a randomly distributed population. This enhanced suppression can significantly reduce the electron scattering optical depth $\tau$, as required by the three-year data from the Wilkinson Microwave Anisotropy Probe (WMAP). We argue that source clustering is likely to similarly boost the importance of a variety of other feedback mechanisms.

Subject headings: cosmology: theory - early Universe - galaxies: high-redshift - evolution
\end{abstract}

\section{INTRODUCTION}

The three-year data on the polarization of the cosmic microwave background from the Wilkinson Microwave Anisotropy Probe (WMAP) satellite have provided an important new constraint on the reionization history of the intergalactic medium (IGM). The WMAP experiment has measured the optical depth $\tau$ to free-electron scattering between us and the recombination epoch at $z \approx 1100$. The result, $\tau=0.09 \pm 0.03$, implies a redshift for instantaneous reionization of $z \approx 11$ (Spergel et al. 2006), and can be used to rule out a tail of significant ionization extending out to higher redshifts. Another constraint on reionization follows from the Gunn-Peterson (GP) troughs detected in the spectra of bright quasars at $z \approx 6$. These GP troughs probe the neutral hydrogen along the line of sight, indicating that the last $\sim 0.1 \%$ of the neutral fraction was rapidly disappearing around this epoch (Fan et al. 2006; Mesinger \& Haiman 2004).

The relatively short reionization history implied by the combination of these constraints requires a strong suppression of ionizing radiation from "minihalos" with virial temperatures $T_{\text {vir }}<10^{4} \mathrm{~K}$ (Haiman \& Bryan 2006). A variety of feedback mechanisms might play a role in this suppression (e.g. Haiman \& Holder 2003). In this paper, we will explore one such feedback mechanism - the suppression of gas infall and star formation in minihalos in photoionized regions - and focus on how the clustering of the ionizing sources enhances this effect.

Many other feedback mechanisms may also play a role in driving the history of reionization. Some (such as the buildup of an X-ray background) may affect the whole IGM more or less uniformly and simultaneously, but most will be localized to within a small fraction of the Hubble distance, at least for

Electronic address: roban@astro.columbia.edu, zoltan@astro.columbia.edu, peng@physics.ucsb.edu some time. Examples of such localized feedback mechanisms are (1) the first generations of stars will enrich the intergalactic medium with metals (Madau et al. 2001; Aguirre et al. 2001), which will affect the formation and evolution of subsequent generations of stars (Tumlinson \& Shull 2000; Bromm et al. 2001; Schaerer 2002; Wyithe \& Loeb 2003); (2) ionizing UV photons can both catalyze the formation of (Ricotti et al. 2002b; Oh \& Haiman 2002; O'Shea et al. 2005), and directly destroy (Haiman et al. 1997a) $\mathrm{H}_{2}$ molecules, which will affect the ability of gas to cool and form structures (Haiman et al. 1997b; Haiman et al. 2000; Ciardi et al. 2000), (3) gas in regions that have been ionized may not be able to settle into the shallow potential wells of small dark matter halos, exacerbating the $\mathrm{H}_{2}$-photodissociating effects of a Lyman Werner background (Haiman et al. 1997b; Mesinger et al. 2006) and increasing the minimum size required for a halo to contain collapsed baryonic structures (Ricotti et al. 2002a,b; Oh \& Haiman 2003).

Since these mechanisms operate over a limited length scale, their effects will depend strongly on the spatial distribution of halos hosting ionizing sources. Numerical simulations are a promising way to address feedback among clustered sources, since they capture the full, three-dimensional relationships among the host halos. However, the dynamic range required to resolve the small minihalos, within a large enough cosmic volume to be representative, remains a challenge, especially in simulations that include radiative transfer (see, e.g. Iliev et al. 2006). Semi-analytical models avoid the problems associated with the large dynamical range; they are also an efficient way to explore parameter space and serve as important sanity checks for more complicated simulations.

We believe that this paper describes the first semi-analytical model to include feedback and clustering simultaneously as a way to suppress ionizing radiation from minihalos. Prior work on the clustering of halos has focused on the problem of de- 
riving analytical expressions for the bias between the distribution of dark matter halos and of mass (Scannapieco \& Barkana 2002); (see also Mo \& White 1996; Scannapieco \& Thacker 2005). These results have been applied to the distribution of minihalos at high redshifts: Haiman et al. (2001) included the bias to account for the effects of minihalo photoevaporation; Iliev et al. (2005) likewise characterized the effect of minihalo clustering on the propagation of ionization fronts through the IGM. Minihalos were considered to be sinks of ionizing photons in both of these works (as well as in Ciardi et al. 2006; Furlanetto \& Oh 2005). In contrast, in the present paper, we examine minihalos as potential sources of ionizing radiation. This means that the radius of influence of our feedback mechanism cannot be simply evaluated at a fixed time-step; instead, it is a function of the entire ionization history of the region, including the history of mergers between ionized regions. The preceding methods cannot be easily be adapted to such a calculation, and we have been driven to employ a novel MonteCarlo means of simulating the coupled history of ionization and feedback around any given point. Our primary conclusion is that, because of their highly clustered distribution, local feedback effects can dramatically suppress star formation in minihalos.

The rest of this paper is organized as follows. In $\S 2$, we describe our model for reionization, extending the formalism of Furlanetto, Zaldarriaga, \& Hernquist (2004) to include an approximate treatment of biased halo distribution and feedback. In $\S 3$, we report our results for the reionization history, and discuss the impact of clustering on the overall suppression of minihalos, on the size distribution of ionized bubbles, and on the optical depth to electron scattering. In $\S 4$, we discuss several limitations and possible extensions and future applications of our model. Finally, in $\S 5$, we summarize our conclusions and the implications of this work.

\section{MODELING REIONIZATION WITH CLUSTERING AND FEEDBACK}

We have chosen to implement the suppression of minihalos in ionized regions (Thoul \& Weinberg 1996; Dijkstra et al. 2004; Oh \& Haiman 2003), both to understand the effects of this particular mechanism, and as an example of localized feedback mechanisms in general. We parameterize the suppression effect by setting different minimum virial temperatures for a halo to contribute to reionization $T_{\mathrm{vir}, \mathrm{min}}$, in ionized and neutral regions:

$$
T_{\mathrm{vir}, \min }=\left\{\begin{array}{l}
10^{2} \mathrm{~K} \text { in neutral regions, } \\
10^{4} \mathrm{~K} \text { in ionized regions. }
\end{array}\right.
$$

We will hereafter refer to halos with virial temperatures between $10^{2} \mathrm{~K}$ and $10^{4} \mathrm{~K}$ as "minihalos", and halos with virial temperatures above $10^{4} \mathrm{~K}$ as "large halos".

Furlanetto, Zaldarriaga, \& Hernquist (2004) (hereafter FZH) have created an elegant, excursion-set-based approach to following the ionized fraction of the Universe, which captures the clustering of ionizing sources. They begin with the assumption that the amount of mass, $M_{\text {ion }}$, that sources within a given halo can ionize, is simply proportional to the total mass of the halo,

$$
M_{\text {ion }}=\zeta M_{\text {halo }},
$$

where $\zeta$ is the "ionization efficiency factor". The ionized fraction $x_{\mathrm{i}}$ of a region is then proportional to the fraction of mass in that region that is contained in collapsed objects,

$$
x_{\mathrm{i}}=\zeta f_{\text {coll }}\left(M, \delta, z, T_{\mathrm{vir}, \min }\right) \text {, }
$$

where $M$ is the mass of the region, $\delta \equiv(\rho-\bar{\rho}) / \bar{\rho}$ is the overdensity of the region, and $z$ is the redshift.

The collapsed fraction in the extended Press-Schechter formalism is:

$$
f_{\text {coll }}\left(M, \delta, z, M_{\min }\right)=\operatorname{erfc}\left[\frac{\delta_{c}(z)-\delta(M)}{\sqrt{2\left[\sigma^{2}\left(M_{\min }\right)-\sigma^{2}(M)\right]}}\right] .
$$

The critical density for collapse is $\delta_{c}=1.686 / g(z)$, where $g(z)$ is the linear growth factor of density perturbations, normalized to unity at $z=0$ (we use the approximation from Carroll et al. 1992). The variance of $\delta(M), \sigma^{2}(M)$, is determined from the power spectrum, which we in turn calculate using the fitting formulae by Eisenstein \& Hu (1999).

We adopt the relation between virial temperature and halo mass given by Barkana \& Loeb (2001):

$$
\begin{aligned}
T_{\text {vir }}(M, z)= & 1.98 \times 10^{4} \mathrm{~K}\left(\frac{M}{10^{8} h^{-1} \mathrm{M}_{\odot}}\right)^{2 / 3}\left(\frac{1+z}{10}\right) \\
& \times\left[\frac{\Omega_{M, 0}}{\Omega_{M}(z)} \frac{\Delta_{\mathrm{c}}}{18 \pi^{2}}\right]^{1 / 3}\left(\frac{\mu}{0.6}\right),
\end{aligned}
$$

where $\Delta_{\mathrm{c}} \equiv 18 \pi^{2}+82\left[1-\Omega_{M}(z)\right]-39\left[1-\Omega_{M}(z)\right]^{2}$, and we set $\mu=1.22$ for $T_{\text {vir }}<10^{4} \mathrm{~K}$ and $\mu=0.59$ for $T_{\text {vir }} \geq 10^{4} \mathrm{~K}$. We solve this expression for $M$ to determine the minimum halo mass, $M_{\min }\left(T_{\mathrm{vir}, \min }, z\right)$, as a function of redshift.

The condition for a region of mass $M$ to be fully ionized is $\zeta^{-1} f_{\text {coll }} \geq 1$. FZH solve this equation for $\delta(M)$ yielding a barrier function $\delta_{x}(M, z)$. The largest mass at which $\delta(M) \geq \delta_{x}(M, z)$ about a particular point is then identified as the mass of the ionized region to which the point belongs. Computing the mass $M$ defined by this identification at different redshifts then translates into the history for the mass of the ionized bubble in which the given point resides. Conversely, the mass function of ionized bubbles at any redshift can be constructed either by Monte Carlo realizations of many $\delta(M)$ trajectories, or from the statistics of the first-crossing distribution of a random walk (for a method of finding the first-crossing distribution for an arbitrarily shaped barrier, see Zhang \& Hui 2005).

The cosmological parameters used throughout this paper are: $h=0.72, \Omega_{M, 0}=0.27, \Omega_{\Lambda}=1-\Omega_{M, 0}, \Omega_{\mathrm{b}, 0}=0.045$, $\sigma_{8}=0.9, n=1$, taken from the best-fit values in the 1 -year WMAP data release (Spergel et al. 2003). For our purposes, the most significant changes in the cosmological parameters in the 3-year WMAP results (Spergel et al. 2006) are the lower values of $\sigma_{8}=0.74_{-0.06}^{+0.05}$ and $n_{s}=0.951_{-0.019}^{+0.015}$. The main effect of both of these changes is to slightly delay structure formation and reionization. This can be compensated for by increasing the efficiency parameter $\zeta$, and it does not affect our primary conclusions about the effect of clustering.

\subsection{Adding feedback}

We next depart from the FZH formalism to incorporate photoionization feedback. Adding feedback means that the barrier $\delta(M)$ at any given redshift can no longer be pre-specified; rather, it will now be a function of the entire ionization history of the region being considered. We therefore must resort to Monte-Carlo simulations to calculate the coupled history of ionization and feedback around any given point.

If we assume that minihalos are randomly distributed and unbiased with respect to the ionized bubbles, then the ionized fraction of a region can be found by integrating the following 

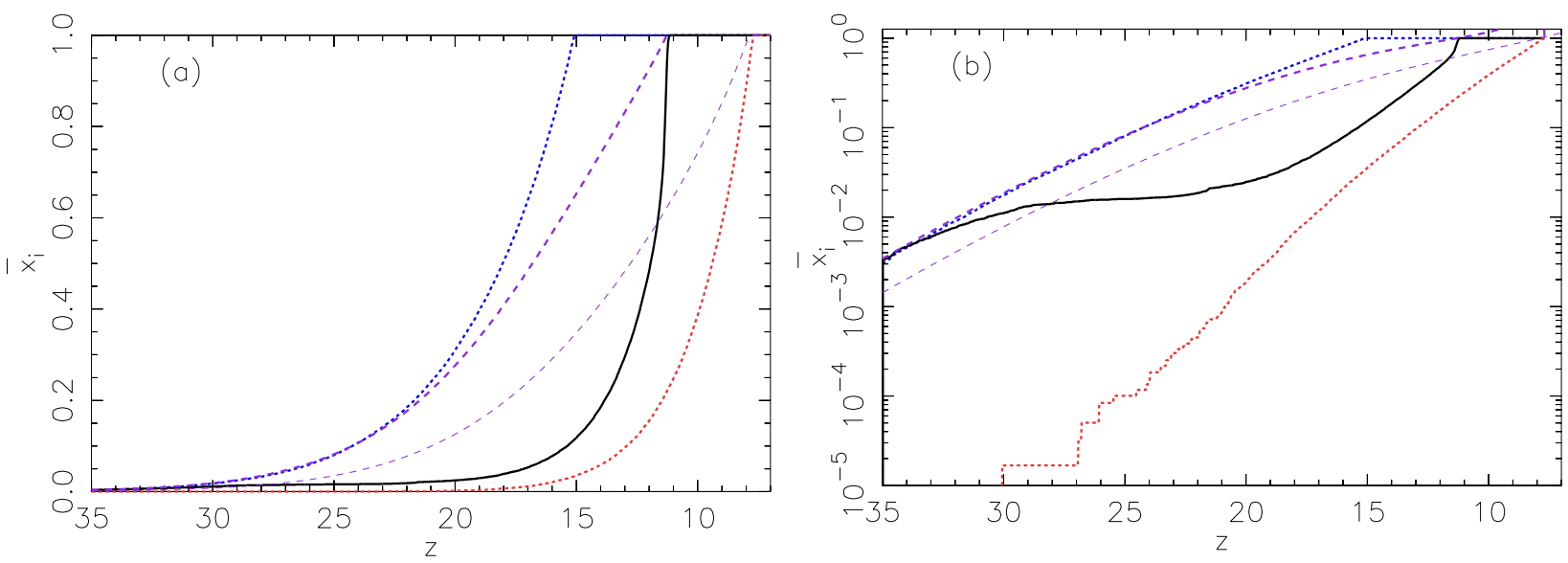

FIG. 1.- The ionization history of the Universe: mean ionized fraction versus redshift. The solid curve is the history with biased feedback. The leftmost short-dashed curve (blue in the color version) includes contributions from all halos, the rightmost short-dashed curve (red) includes only large halos, and the upper long-dashed curve (purple) includes unbiased feedback. All of the above use $\zeta=12$. The light-weight long-dashed curve (purple) is an unbiased feedback calculation with a lowered efficiency factor, $\zeta=5$. Comparing the lower unbiased feedback curve with the biased feedback curve demonstrates that minihalo clustering must have a large effect on the early stages of reionization even if the reionization redshift is assumed to be known.

simple differential equation, starting from high redshift:

$$
\frac{d x_{\mathrm{i}}}{d z}=\frac{d x_{\mathrm{i}, \text { large }}}{d z}+\left(1-x_{\mathrm{i}}\right) \frac{d x_{\mathrm{i}, \text { mini }}}{d z}
$$

where $d x_{\mathrm{i}, \text { large }} / d z$ is the contribution to ionizing the region from large halos and $d x_{\mathrm{i}, \mathrm{mini}} / d z$ is the contribution from minihalos:

$$
\begin{aligned}
& \frac{d x_{\mathrm{i}, \text { large }}}{d z} \equiv \zeta \frac{d f_{\text {coll }}}{d z}\left(T_{\text {vir }}>10^{4} \mathrm{~K}\right) \\
& \frac{d x_{\mathrm{i}, \text { mini }}}{d z} \equiv \zeta \frac{d f_{\text {coll }}}{d z}\left(10^{2} \mathrm{~K}<T_{\text {vir }}<10^{4} \mathrm{~K}\right)
\end{aligned}
$$

Note that these expressions follow FZH and ignore recombinations (we will discuss this issue in $\S 4.1$ below). Applying these equations to the whole Universe (i.e., to a region of mass $M \rightarrow \infty, \delta=0$ ) gives us the reionization history of the Universe in the absence of any clustering.

The efficiency factor is chosen to be $\zeta=12$, unless stated otherwise. This factor can be thought of as the product

$$
\zeta \equiv f_{\text {esc }} f_{*} N_{\gamma / b}\left(1+n_{\text {rec }}\right)^{-1}
$$

where $f_{\text {esc }}$ is the fraction of ionizing radiation that escapes from halos, $f_{*}$ is the fraction of baryons that end up in stars, $N_{\gamma / b}$ is the number of ionizing photons produced per stellar baryon, and $n_{\text {rec }}$ is the average number of times a hydrogen atom recombines. As an example, $\zeta=12$ could be obtained by adopting the reasonable choices of $f_{\mathrm{esc}}=0.1, f_{*}=0.2, N_{\gamma / b}=$ $4200, n_{\text {rec }}=6$. We ignore the dependence of $n_{\text {rec }}$ on bubble size, an approximation which becomes inaccurate toward the tail end of reionization (Furlanetto \& Oh 2005). We discuss this issue further in $\$ 4.1$.

The integration is performed numerically from $z=35$ to $z=7$ with step size $d z=0.02$. All distances and densities specified in this paper are in Lagrangian comoving coordinates. Lagrangian coordinates are appropriate for our problem since we care more about the column density between two points (which is directly related the required number of ionizing photons) than about their physical separation.

Figure 1 shows the evolution of the ionized fraction in the above toy model. The figure displays the history with only large halos allowed to contribute (rightmost short-dashed, red, curve), with all (i.e. both large and mini-) halos contributing (leftmost short-dashed, blue, curve), and with minihalos suppressed by unbiased feedback (upper long-dashed, purple, curve). The first two curves will bound the reionization history with feedback, since they represent the most extreme cases. As expected, the unbiased feedback history begins following the all-halo curve, then lags behind as the ionized fraction increases and minihalos are suppressed.

\subsection{Capturing clustering}

In order to capture the biased distribution of the minihalos with respect to the ionized bubbles, we begin by synthesizing random walk trajectories representing the overdensity $\delta(M)$ around a random point in space averaged over spheres of mass $M$. Figure 2a shows such profiles. The overdensity tends towards zero as $M$ gets large, since density fluctuations average out in very large spheres.

We then divide the trajectory up into thin spherical shells and numerically integrate equation (6) for each shell separately. In this paper shells are logarithmically spaced from $10^{4}-10^{18} \mathrm{M}_{\odot}$ with thickness $d \log \left(M / \mathrm{M}_{\odot}\right)=0.015$. Shells that overproduce ionizing photons are allowed to contribute to the ionization of the outside neighboring shell (this is calculated recursively to allow extra photons to spill outward until consumed). For the purpose of finding the mass of the ionized bubble surrounding the central point, as defined above, the ionized mass is still associated with the shell from which the ionizing photons originated.

The total mass ionized by sources within a sphere of size $M$ is the sum of the contribution from all interior shells,

$$
M_{\text {ion }}(M)=\sum_{\mathrm{M}^{\prime}<\mathrm{M}} M_{\text {ion,shell }}\left(M^{\prime}\right) .
$$

As in the FZH formalism, a point is said to belong to an ionized bubble of mass $M$ such that $M$ is the largest mass for which $M_{\text {ion }}(M) \geq M$.

Figure $2 \mathrm{~b}$ shows the mass histories corresponding to the four density profiles shown in Figure $2 \mathrm{a}$ for the all-halo, large-halo-only, and biased feedback cases. As was found in Furlanetto \& Oh (2005), the growth of the region proceeds primarily by large jumps in mass, corresponding to "major mergers", wherein a point is engulfed by a neighboring, larger 

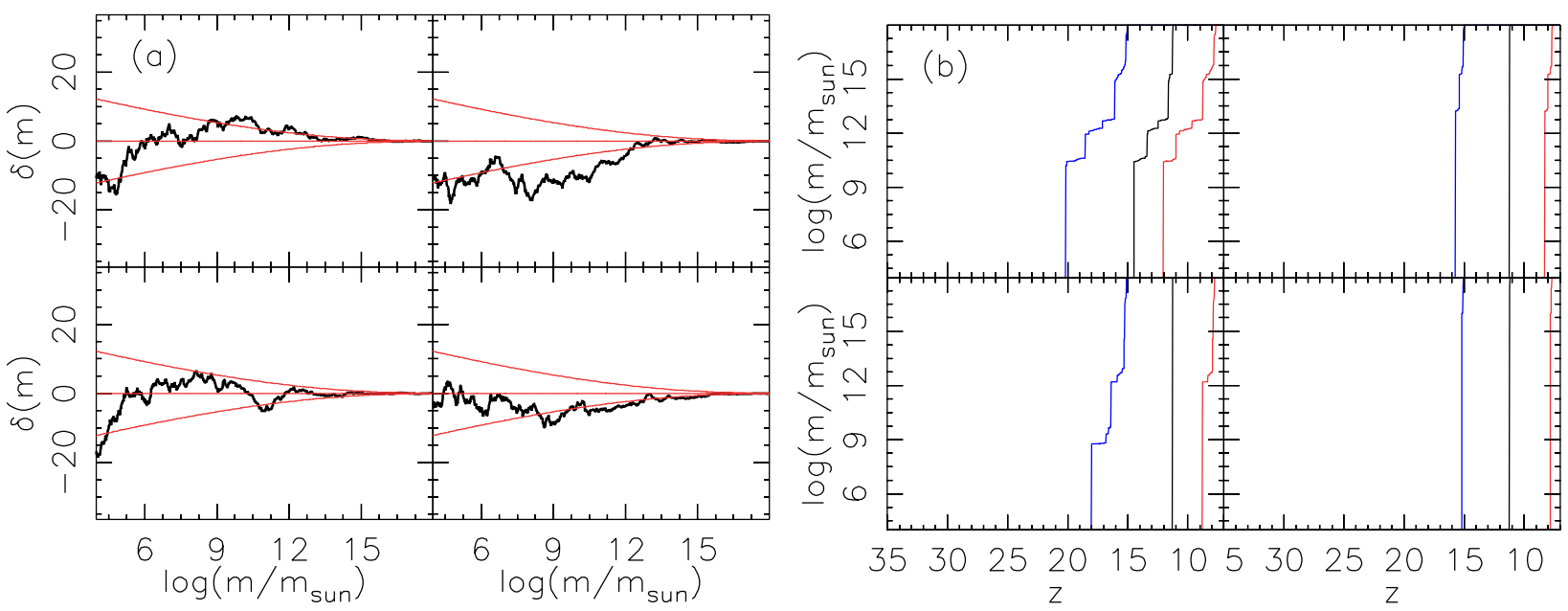

FIG. 2.- Examples of the density profile (a) and the corresponding HII region mass history (b) about four randomly chosen points. Heavy (black) lines in (a) show the mean overdensity in a spherical region versus the mass of the region, extrapolated linearly to the present. Light (red in the color version) curves mark the $\pm 1 \sigma$ level for the density fluctuations. In (b) the HII region mass to which each point belongs is plotted against redshift for three cases: (left) all (mini- and large) halos contribute to reionization, (center) feedback is turned on, and (right) only large halos contribute.

HII region. The mass of a merger product is determined by where a peak occurs in the $\delta(M)$ trajectory, so many of these jumps are repeated in each history at the same mass, but at different redshifts. This explains why the trajectories in the three different cases in Figure $2 b$ appear to be nearly identical copies of one another, shifted in redshift.

Note that this prescription only partially captures the clustering of minihalos. Because of the division into concentric shells, the radial dimension of the clustering around each point is well captured, while the clustering of halos in the tangential direction, within each thin shell, is ignored. The model, therefore, performs best while ionized bubbles are small; otherwise, the effects of clustering are underestimated. This means that the predicted minihalo suppression is a lower limit, or, equivalently, the predicted bubble masses and ionized fractions upper limits.

The probability that a randomly selected point in space will land in an ionized bubble of mass $M$ to $M+d M$ is equal to the fraction of the total volume (or mass) of the IGM occupied by such bubbles, $f(M, z)=d x_{\mathrm{i}}(M, z)$, where $f(M, z)$ is the fraction of bubble mass histories falling between $M$ and $M+d M$ at redshift $z$. The ionized fraction of the Universe is then

$$
\bar{x}_{\mathrm{i}}(z)=\sum_{m} f(M, z)
$$

We have performed the above calculation for an ensemble of $N_{\text {total }}=60,000$ realizations of the density profile; this implies that we can resolve a minimum global ionization fraction of $\bar{x}_{\mathrm{i}} \sim 1 / N_{\text {total }} \sim 10^{-5}$.

\section{RESULTS}

\subsection{Universal ionized fraction}

Figure 1 shows the reionization history computed from our models. In addition to the unbiased feedback, all-halos, and large-halos-only cases discussed previously, the figure also displays the result in the case with biased feedback (thick solid black curve). Adding clustering dramatically reduces the ionized fraction at high redshift. The biased history departs from the unbiased history before $\bar{x}_{\mathrm{i}}=0.01$ and reaches a maximum fractional suppression relative to the unbiased case of a factor of 11 , when $\bar{x}_{\mathrm{i}, \text { bias }}=0.03$ vs. $\bar{x}_{\mathrm{i}, \text { unbias }}=0.33$ at $z=19.2$, and a maximum absolute difference of 0.56 when $\bar{x}_{\mathrm{i}, \text { bias }}=0.22$ vs. $\bar{x}_{\mathrm{i} \text {,unbias }}=0.76$ at $z=13.6$.

The figure shows that the unbiased and biased reionization histories (which have the same efficiency, $\zeta=12$ ) reach percolation at the same redshift; i.e. they both meet at $\bar{x}_{\mathrm{i}}=1$ at $z \sim 11.1$. This is an artifact of the modeling procedure, and is not physical. It arises because the largest, outermost shells in each density profile will always tend to have overdensities very close to $\delta=0$, so their ionization histories will match that of an unbiased Universe, and will therefore be fully ionized when the unbiased Universe is fully ionized. In reality, feedback would always delay percolation, as long as the contribution of minihalos to the ionization is not negligible $\left(d f_{\text {coll,mini }} / d z \gtrsim d f_{\text {coll,large }} / d z\right.$; see Fig. 7 below $)$. We also emphasize that our neglect of clustering in the tangential direction within each shell will always increase the universal ionized fraction over what it would be if the clustering in the tangential direction was also included. Therefore, the difference between the unbiased and biased histories is a lower limit, and our conclusion that clustering significantly increases minihalo suppression is conservative and robust.

Since observations of Gunn-Peterson troughs in quasar spectra constrain the end of reionization to $z_{\mathrm{EOR}} \approx 6$, and since the value of the efficiency parameter $\zeta$ is uncertain, a better comparison might be between unbiased and biased trajectories with fixed $z_{\mathrm{EOR}}$, rather than fixed $\zeta$. The artifact mentioned above means that our biased feedback model becomes unreliable near percolation, making such an exact comparison impossible. However, we know that our biased feedback calculation together with the large-halo-only curve constitute upper and lower bounds on the true ionized fraction in the biased feedback case. Therefore, if we calculate an unbiased feedback history with an efficiency factor lowered (we find, to $\zeta=5$ ) so that it reaches full ionization at the latest possible redshift $\left(z_{\mathrm{EOR}} \approx 7.7\right.$, matching the large-halos-only case), then it, together with the $\zeta=12$ unbiased feedback curve, constitute commensurable upper and lower bounds.

In other words, in the context of a fixed $z_{\mathrm{EOR}}$, the minimum possible effect at high redshift of adding clustering is shown by the difference between the unbiased feedback curve with $\zeta=5$ and the biased feedback curve with $\zeta=12$. The 
comparison of the thick solid (black) and the light-weight, long-dashed (purple) curves in Figure 1 clearly demonstrates that minihalo clustering has a significant effect on the highredshift tail of reionization. In Figure $1 \mathrm{~b}$, which plots the logarithm of the ionized fraction versus redshift, it can be seen that the biased feedback curve initially follows the all-halo curve, as expected. However, at $z \sim 30$, the ionized fraction reaches a plateau, indicating that nearly all minihalo formation is suppressed (see the next subsection); the ionized fraction does not begin to increase again until the contribution from large halos becomes significant.

\subsection{Ionized bubble sizes}

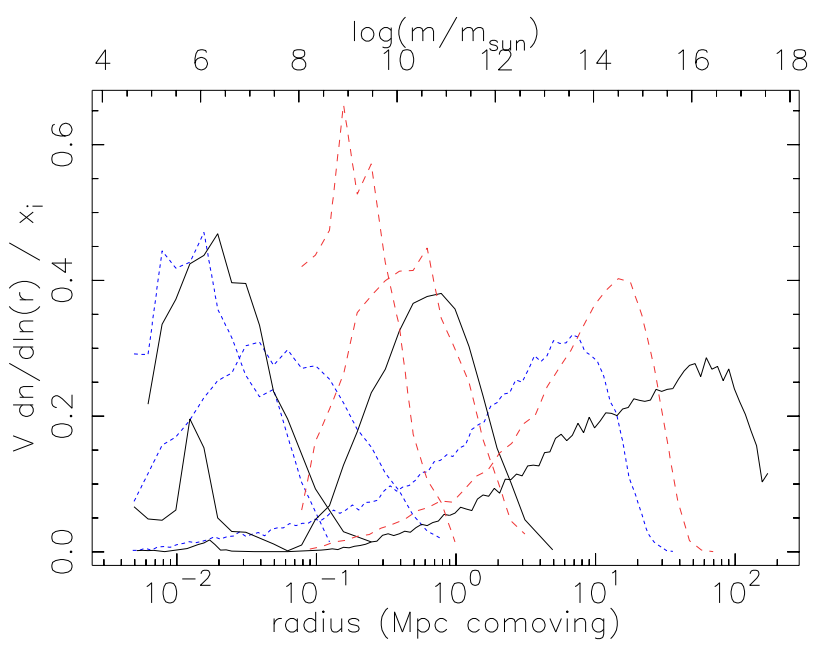

FIG. 3.- The size distribution of HII regions shown for $\bar{x}_{\mathrm{i}}=0.01,0.1,0.75$ (left to right in each set). The solid curves include biased feedback, the shortdashed curves include all halos and the long-dashed curves are for large halos only. The comoving bubble radius is indicated on the bottom axis, and the equivalent mass on the top axis. The redshifts corresponding to the ionized fractions listed above are $z=31.7,24.3,16.3$ with all halos, 30.7.15.3,11.4 with biased feedback, and 17.3,12.9,8.4 with large halos only.

In order to better understand the results in the previous section, we here examine the size-distribution of the ionized bubbles. The fraction of points lying in bubbles between mass $M$ and $M+d M$ is

$$
f(M, z)=d \bar{x}_{\mathrm{i}}(z)=V(M) \frac{d n_{\mathrm{HII}}}{d M} d M
$$

where $V(M)$ is the volume of a region of mass $M$, and $d n_{\mathrm{HII}} / d M$ is the number density of ionized bubbles per unit mass between $M$ and $M+d M$. We solve this equation to find the mass distribution of the bubbles, given the histogram of HII region masses derived from our ensemble of density profiles. Figure 3 plots the size distribution of ionized bubbles, normalized by the average ionized fraction of the Universe (after FZH), $V(M) d n_{\mathrm{HII}} / d \ln (r) \bar{x}_{\mathrm{i}}^{-1}$, for $\bar{x}_{i}=0.01,0.1,0.75$. This function is chosen so that the area under a segment of the curve (in log space) is equal to the fraction of ionized gas contained in bubbles in that radius (or mass) interval.

The leftmost set of curves in the figure shows that when there is very little ionized gas $\left(\bar{x}_{\mathrm{i}}=0.01\right)$, it is mostly contained in bubbles around individual halos. Bubble sizes are therefore close to $M_{1} \equiv \zeta M_{\min }(z)$, the size of a bubble around a single, minimum-mass halo. For minihalos, $M_{1} \sim 10^{5} \mathrm{M}_{\odot}$, while for large halos $M_{1} \sim 10^{8} \mathrm{M}_{\odot}$. The equivalent comoving radii are $\sim 10^{-2} \mathrm{Mpc}$ and $\sim 10^{-1} \mathrm{Mpc}$.
At the intermediate value $\bar{x}_{\mathrm{i}}=0.1$, the feedback case has developed a double-peaked distribution. The lower peak corresponds to bubbles close to $M_{1} \equiv \zeta M_{\min }(z)$ for minihalos, while the upper peak corresponds to larger bubbles caused by source clustering. Note that this bimodal distribution is unique to the biased feedback scenario: since the minihalo contribution in ionized bubbles is greatly attenuated, the number of isolated bubbles of mass $M_{1}$ is boosted. By the time reionization is nearing completion, $\bar{x}_{\mathrm{i}}=0.75$, the lower-radius peak has nearly disappeared and the feedback case has a peak radius that exceeds the other two cases. It is easy to understand why the bubble size in the feedback case exceeds that of the all-halos case: the contribution of minihalos is attenuated and the larger bubble size reflects the stronger clustering of the larger halos. Similar effects emerge when one imposes a mass-dependent ionizing efficiency (Furlanetto et al. 2006). Note that, in fact, at $\bar{x}_{i} \geq 0.4$, the mean bubble size in the feedback case even exceeds that in the large-halos-only case. We caution, however, that this result may be unphysical: as discussed above, our results become quantitatively unreliable at low redshift. In particular,the mean ionized fraction of $\bar{x}_{i} \approx 0.4$ is reached at the redshift below which the minihalo contribution starts to increase (see Fig. 7), suggesting that our minihalo suppression becomes only a lower limit below these redshifts. It is not clear whether a more accurate treatment, with more minihalo suppression, would further increase or decrease the mean bubble size. Finally, we note that that in the biased feedback case, the distribution of bubble sizes broadens as reionization proceeds, whereas without feedback the bubble size distribution narrows, for fairly general reasons (Furlanetto et al. 2006); this may be another signature of the unphysical increase in minihalo contribution.

To gain further intuition, we explore how the mean bubble size and the number density of bubbles evolves with redshift in each case. We define a characteristic bubble size as the mean mass-weighted bubble radius:

$$
\bar{r}=\frac{1}{\bar{x}_{\mathrm{i}}} \int r V(r) \frac{d n_{\mathrm{HII}}}{d r} d r .
$$

When the evolution of the characteristic size is considered in redshift (Figure 4a), $\bar{r}$ with feedback is intermediate between the all-halo and large-halo-only cases, both in the very beginning (at $z \gtrsim 30$, when isolated bubbles dominate), and also later (at $z \lesssim 18$, when enough large halos have formed to drive the distribution). However, when $\bar{r}$ is considered against the ionized fraction $\bar{x}_{\mathrm{i}}$ (Figure $4 \mathrm{~b}$ ), the radius in the feedback case quickly exceeds that of both "extremes", although as noted above, the mild excess above the large halo only case may not be physical. Note that all three curves should approach the Hubble radius as $\bar{x}_{\mathrm{i}}$ approaches unity. The unphysical convergence at $M=10^{18} \mathrm{M}_{\odot}$ occurs because this is the largest mass scale included in the numerical modeling. For reference, in Figure 4, we also show the correlation lengths, defined by $b\left(M_{1}\right) b\left(M_{2}\right) \xi(r)=1$, where $b(M)$ is the linear bias of halos (Sheth \& Tormen 1999), and $\xi(r)$ is the correlation function of mass.

The total number density of ionized bubbles, $n_{\mathrm{HII}}=$ $\int\left(d n_{\mathrm{HII}} / d r\right) d r$, is plotted versus redshift and ionized fraction in Figure 5. Comparing this to Figure 4 allows us to describe the growth of HII bubbles during the reionization process.

During the first phase of reionization, most of the ionized gas is contained in small bubbles around isolated halos, or groups of a few halos. The increase in the bubble size is mostly due to the formation of new, larger halos, and the num- 

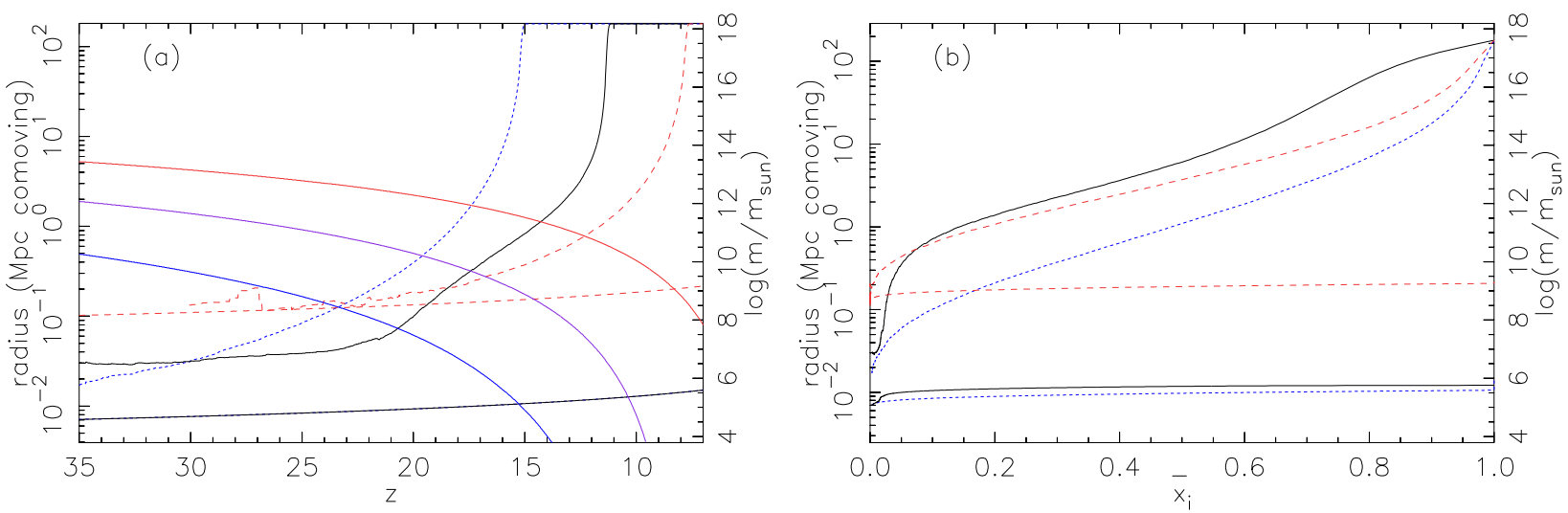

FIG. 4.- The mean HII region size versus redshift (a) or versus Universal ionized fraction (b) for the large-halo-only (long-dashed), all-halo (short-dashed), and biased feedback (solid) cases. Also shown (nearly-flat curves) is the minimum size $M_{1}$ for an isolated bubble around a minimum-mass halo for each case. The three solid curves that curve downwards at low redshift are (from top to bottom) the correlation lengths for large halos, the cross-correlation length between large and minihalos, and the correlation length for small halos.

ber of HII regions climbs steadily. This phase ends when the characteristic bubble size reaches the correlation length of the relevant halo population. At this point, the second phase begins. Bubbles start to merge rapidly, and the growth of the characteristic bubble size is now primarily due to those mergers. The number of HII regions declines steadily as the bubble sizes grow larger and larger. Reionization is complete when the bubble size becomes formally infinite.

In the feedback case, the transition to the second phase occurs at a much smaller ionized fraction than in the other two cases (note the early peak in Figure $5 b$ ). At $z \approx 24$ in the all-halo case, the bubble size exceeds the correlation length, but this is delayed by minihalo suppression in the feedback case until $z \approx 20$. By this time there are more bubbles (even though the ionized fraction is lower), because more isolated halos have had time to form in slightly less-dense regions, and these bubbles have not yet merged away.

As a result, we find that minihalo suppression slows the growth of the bubbles and delays their mergers, while allowing more individual bubbles to form. Because the bubbles are smaller, they can be more tightly "packed", i.e. their separations are smaller, so when they do begin to overlap, a very rapid period of bubble growth by mergers ensues $(z \approx 22$ to $z \approx 20$ ). Because the mergers occur so rapidly (and because of high minihalo suppression), the ionized fraction grows very little, while the bubble size grows by over an order of magnitude (note the sharpness of the peak in Figure $5 \mathrm{~b}$ and the rapid rise in radius in Figure 4b). This means that the mean bubble size for the feedback case ends up very high, for any given ionized fraction at $\bar{x}_{\mathrm{i}} \gtrsim 0.05$.

\subsection{Mean bias factor}

Our main result above can be summarized as follows: a much larger fraction of minihalos are suppressed when their clustering is included, relative to the value of $\bar{x}_{\mathrm{i}}$ expected if the minihalos were randomly distributed. Here we compare the "boost" in the fraction of suppressed halos we find to naive expectations, using a linear halo-bias model.

Let us first assume that the average ionized fraction of the Universe evolves according to this differential equation,

$$
\frac{d \bar{x}_{\mathrm{i}}}{d z}=\frac{d \bar{x}_{\mathrm{i}, \text { large }}}{d z}+\left(1-\bar{B}(z) \bar{x}_{\mathrm{i}}\right) \frac{d \bar{x}_{\mathrm{i}, \text { mini }}}{d z},
$$

where $\bar{B}(z)$ is the effective "boost", i.e. the enhancement in the number of minihalos forming in ionized regions, relative to $\bar{x}_{\mathrm{i}}$, due to their biased distribution. Since we know each $d \bar{x}_{\mathrm{i}} / d z$ and $\bar{x}_{\mathrm{i}}$ from our simulation runs, this equation serves as a definition of $\bar{B}(z)$. Figure 6 plots this factor, calculated from the biased feedback ionization history using

$$
\bar{B}(z)=\frac{1}{\bar{x}_{\mathrm{i}}}\left(1-\frac{d \bar{x}_{\mathrm{i}} / d z-d \bar{x}_{\mathrm{i}, \text { large }} / d z}{d \bar{x}_{\mathrm{i}, \text { mini }} / d z}\right) .
$$

Because the $d \bar{x}_{\mathrm{i}} / d z \geq d \bar{x}_{\mathrm{i}, \text { large }} / d z$, we know that $\bar{B} \geq \bar{x}_{\mathrm{i}}^{-1}$. Note that $\bar{B}=\bar{x}_{\mathrm{i}}^{-1}$ represents the limiting case in which all minihalos are suppressed.

Figure 6 shows that at the very beginning of reionization, the enhancement is relatively low (much less than $\bar{x}_{\mathrm{i}}{ }^{1}$ ) since few bubbles have grown large enough to encompass more than one halo. However, the boost factor quickly climbs to $\bar{B}(z) \approx \bar{x}_{\mathrm{i}}^{-1} \sim 50$, and at $z \lesssim 28$, nearly all minihalos are suppressed. This corresponds to the plateau of the solid curve in Figure 1b, and the epoch of rapid mergers discussed in the previous subsection. The enhancement subsequently decreases as the ionized fraction increases and the ionized regions are sampling regions whose density is closer to the global average. Values of $\bar{B}(z)<1$ are artifacts of the model, caused by the unphysically rapid end of reionization discussed in $\S 3.1$ above.

Naively, we might expect that the enhancement factor would be roughly equal to the enhancement in the average number of halos within a bubble radius of an average halo,

$$
B_{\text {neighbor }}=\frac{3 b\left(M_{1}\right) b\left(M_{2}\right)}{\bar{r}^{3}-r_{\min }^{3}} \int_{r_{\min }}^{\bar{r}} r^{2} \xi_{m}(r) d r,
$$

where $b(M)$ is the bias of halos of mass $M, \xi_{m}(r)$ is the mass correlation function, and $r_{\min }$ is a minimum radius chosen to exclude overlapping halos (though the calculation is quite insensitive to its exact value). Figure 6 shows this enhancement for large halos near large halos, large halos near minihalos, and minihalos near minihalos (using the minimum masses of each halo type). At the earliest stages of reionization the lowest curve - the enhancement in the number of minihalos within a bubble radius of an average minihalo - should be directly applicable. The figure, however, shows that this underestimates the actual bias enhancement by nearly an order of magnitude.

Several effects may contribute to this interesting disparity. First, we are interested in small scales (the ionized mass is 

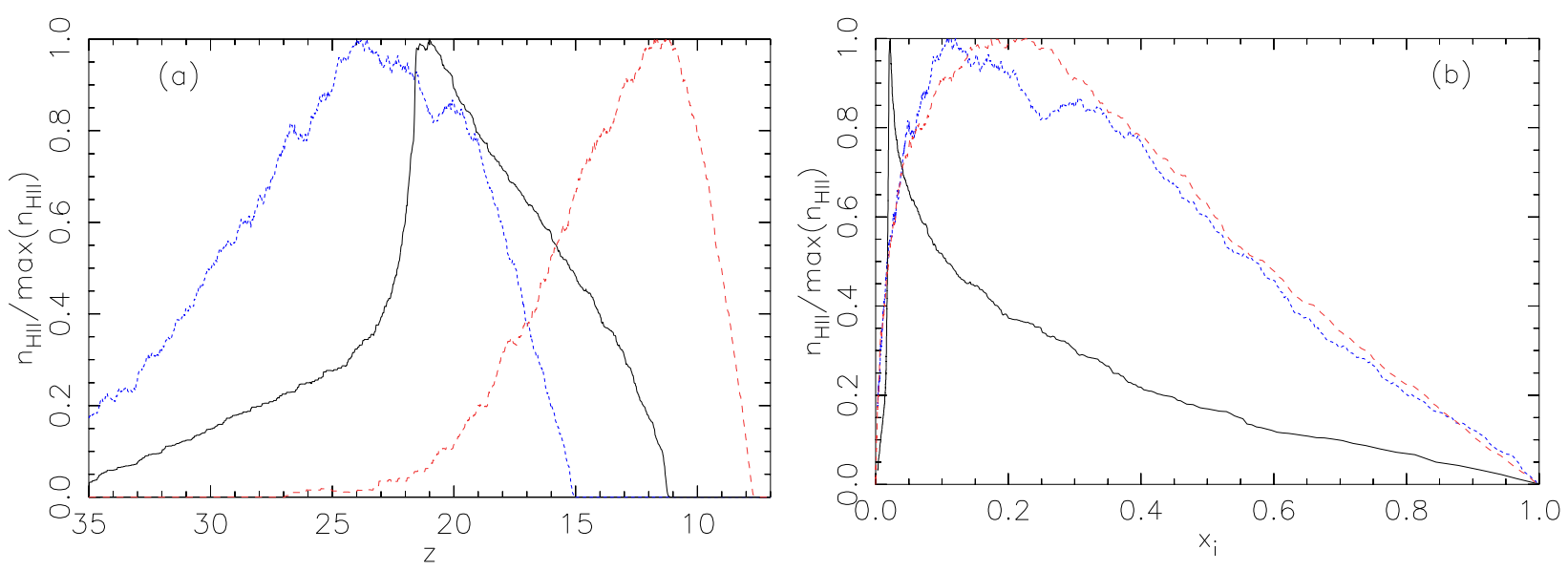

FIG. 5.- The comoving number density of HII regions versus redshift (a) or versus Universal ionized fraction (b) for the large-halo-only (long-dashed), all-halo (short-dashed), and feedback (solid) cases. The plots are normalized by the maximum number density for each case, which is $1.0 \times 10^{4} \mathrm{Mpc}^{-3}$ with all halos, $1.5 \times 10^{4} \mathrm{Mpc}^{-3}$ with biased feedback, and $2.8 \mathrm{Mpc}^{-3}$ with large halos only.

only 12 times the halo mass), where using the linear power spectrum, as we did in equation (16), will underpredict the clustering. As shown by Iliev et al. (2003), the effects of non-linear bias can boost the clustering of minihalos at high redshift on small scales substantially over the standard linear result (for instance, at $z \sim 20$, when our typical bubble size is $\leq 0.1 \mathrm{Mpc}$, Fig. 2 of Iliev et al. (2003) shows that non-linear bias can exceed the standard linear result by an order of magnitude). They show that a non-linear analytic technique based on extended Press-Schechter theory provides an accurate match to numerical simulations; our excursionset based formalism, although different in detail, should likewise capture non-linear bias. Such effects could in principle be largely responsible for the difference between our results and equation (16), although the overall contribution is difficult to assess since volume-exclusion effects will reduce clustering on small scales and will compensate somewhat for our neglecting nonlinear effects. It clearly dominates at the beginning of reionization, when most bubbles have a typical size $M_{1} \equiv \zeta M_{\min }(z)$. Second, equation (16) gives the increase in the number of neighbors centered around a single halo, whereas in our case, the ionized region is already known to contain several halos. Third, equation (16) averages the bias over all halos at a given redshift; in our case, we are interested in the number of new halos forming around a set of halos that had formed earlier. Fourth, the bubble-size distribution in the feedback case always has a tail extending to small sizes, so using the mass weighted mean radius $\bar{r}$ will underestimate the bias; in principle, one should integrate over the distribution of bubble sizes.

In summary, we conclude that given all the caveats and complications mentioned above, either a tailored semianalytic approach or a naive estimate based on linear theory will both give misleading underestimates of the impact of clustering.

\subsection{Minihalo suppression and electron scattering optical depth}

In Figure 7, we show the fraction of the reionization contributed by minihalos as a function of redshift in the biased and unbiased feedback cases. For the reasons discussed previously, the minihalo fraction calculated here for the biased feedback case is an upper limit. In particular, the apparent up-
TABLE 1

OPTICAL DEPTH AND END-OF-REIONIZATION REDSHIFT FOR EACH SCENARIO

\begin{tabular}{rrrrrr}
\hline \hline Scenario: & all & unbiased & unbiased & clustered & large only \\
\hline & & & & & \\
$\zeta$ & 12 & 12 & 5 & 12 & 12 \\
$\tau$ & 0.21 & 0.20 & 0.14 & 0.12 & 0.08 \\
$z_{\text {EOR }}$ & 15.1 & 11.1 & 7.8 & 11.1 & 7.7 \\
\hline
\end{tabular}

turn of the minihalo fraction at low redshift is unphysical. The most conservative conclusion is that less than $60 \%$ of the full ionization would be contributed by minihalos in a more exact treatment of clustered feedback. It is likely, however, that in the absence of the unphysical upturn, the minihalo contribution would fall significantly below this value at $z \lesssim 15$.

Finally, in light of the recent measurement in the three-year WMAP data, it is interesting to compute the impact of minihalo suppression on the optical depth $\tau$ to electron scattering. We compute the value of $\tau$ using the evolution of the ionized fraction of hydrogen in our models. We ignore additional electrons from ionized Helium (if the fraction of singly ionized helium would track that of hydrogen, $\tau$ would increase by $\sim 8 \%$ ).

The optical depths for each history are reported in Table 1. We naturally find the earliest ionization in the no feedback (all-halos) case, so this limiting model has the highest optical depth. With unbiased feedback, reionization is delayed, and we find a slightly lower optical depth. The lower efficiency unbiased case has even lower optical depth. And our history with clustered feedback results in lower optical depth than any other scenario, except the case in which we eliminate all minihalos. Note that since the biased feedback case is an upper limit on the ionized fraction and reaches $\bar{x}_{\mathrm{i}}=1$ artificially early, the calculated optical depth is also an upper limit in this case. On the other hand, the optical depth in the large-halo-only case constitutes a lower limit to the true biased feedback case.

In conclusion, Table 1 shows that clustering reduces the optical depth from somewhere in the range of $0.14<\tau<0.20$ 
to somewhere in the range of $0.08<\tau<0.12$.

\section{DISCUSSION}

\subsection{Caveats}

Obviously many simplifications must be made to construct a semi-analytical model of a process as complicated as reionization. We have already discussed the fact that our results for feedback with clustering constitute an upper limit on the ionized fraction due to the averaging of the suppression effect over spherical shells and the dominance of large shells when the bubbles are large. However, we only start to see obviously unphysical effects $(\bar{B}<1$, or an increasing minihalo contribution to reionization) at $\bar{x}_{\mathrm{i}} \gtrsim 0.4$ or $z \lesssim 12$. We expect that a more exact treatment that incorporates feedback with a threedimensional treatment of clustering would stay close to this upper limit prior to this redshift.

Another important oversimplification is that we have ignored recombinations (except in a very rough way in the $\zeta$ factor). In particular, we ignore the scale dependence of the recombination rate; as the bubbles get bigger and the mean free path of ionizing photons increases, the gas in the IGM is likely ionized up to larger overdensities, and the importance of recombinations will increase. The omission of recombination may also be important in the "stalling" regime we find occurs at high redshift $z \gtrsim 20$ : since there are few ionizing photons being produced and the ionized bubbles are in relatively dense regions, recombinations would likely lower the ionized fraction. Furlanetto \& Oh (2005) describe a method for including recombinations in the $\mathrm{FZH}$ formalism, which might be adapted for use in this model. Although their results suggest that recombinations are only important in the very late stages of reionization, the larger bubble sizes produced in the feedback case may make recombinations important somewhat earlier. However, since the late-time behavior of our biased feedback model when $\bar{x}_{i} \rightarrow 1$ is in any case unreliable (as discussed in $\S 2$ ), we have foregone this extra complication in the present paper.

Furlanetto et al. (2006) and Cohn \& Chang (2006) have explored several generalizations of the FZH formalism, including the use of a more suitable halo mass function, stochastic fluctuations in the halo distribution, a mass-dependent efficiency factor $\zeta(M)$, and the history of halo mergers within ionized bubbles. Their results suggest that the first two modifications would have relatively minor effects on our results. A mass-dependent efficiency, or associating sources with halo mergers, however, could both change the importance of minihalo suppression, since it could change the relative contributions of minihalos and large halos to reionization. In particular, if the efficiency of ionizing photon production in minihalos were reduced enough (by internal feedback, for instance), minihalo suppression in ionized regions would become unimportant.

Our specific assumptions about minihalo suppression are also somewhat idealized. For instance, there will clearly not be a sharp cut-off of minihalo formation at precisely $10^{4} \mathrm{~K}$.
There is also some uncertainty about the exact value of the minimum virial temperature, which may have some environmental dependence, in any case. Changing the dividing line between minihalos and large halos will change their relative populations and affect the precise reionization history, but small changes should not affect the importance of minihalo suppression as a feedback mechanism in general.

\subsection{Possible extensions}

There are several extensions to the formalism presented here that might be profitably explored in the future, such as including a mass-dependent efficiency factor, $\zeta(M)$, and in particular using different efficiencies for large and minihalos. There are possibilities for improving the performance of the model at high $\bar{x}_{i}$, which would allow us to better constrain the end-of-reionization redshift and the optical depth, or, more importantly, to calculate $\tau$ under various assumptions given a fixed $z_{\mathrm{EOR}}$. As mentioned above, our formalism can be easily adapted to treat a variety of feedback mechanisms other than minihalo suppression; our results suggest that the impact of these other feedback mechanisms will generically be increased by the presence of clustering, as well. We plan to apply the formalism discussed here to address the enrichment of the IGM with metals in a future paper.

\section{CONCLUSIONS}

Using a unique semi-analytical technique, we have demonstrated that minihalo suppression in ionized regions is enhanced by clustering, and that such enhanced suppression substantially reduces the high-redshift tail of reionization. Other feedback mechanisms may be similarly enhanced by clustering, since feedback process tend to operates over a limited distance, especially when sources are young.

The compressed ionization history produced by minihalo suppression reduces the optical depth $\tau$ compared to cases without clustering or without feedback. The reduction in $\tau$ occurs both with fixed ionization efficiency factor $\zeta$ and with fixed end-of-reionization redshift $z_{\mathrm{EOR}}$.

Haiman \& Bryan (2006) have shown that substantial minihalo suppression is necessary to simultaneously meet the constraints imposed by Gunn-Peterson troughs on $z_{\text {EOR }}$ and by the value of $\tau$ measured in the three-year data of the WMAP experiment. We conclude that a simple feedback mechanism — such as preventing the formation of minihalos in ionized regions - can produce such suppression when the highlyclustered distribution of these sources is taken into account.

ZH acknowledges partial support by NASA through grants NNG04GI88G and NNG05GF14G, by the NSF through grants AST-0307291 and AST-0307200, and by the Hungarian Ministry of Education through a György Békésy Fellowship. SPO acknowledges NSF grant AST 0407084 and NASA grant 05-ATP05-115 for support.

\section{REFERENCES}

Aguirre, A., Hernquist, L., Schaye, J., Katz, N., Weinberg, D. H., \& Gardner, J. 2001, ApJ, 561, 521

Barkana, R., \& Loeb, A. 2001, Physics Reports, 349, 125

Bromm, V., Kudritzki, R. P., \& Loeb, A. 2001, ApJ, 552, 464

Carroll, S. M., Press, W. H., \& Turner, E. L. 1992, ARA\&A, 30, 499

Ciardi, B., Ferrara, A., \& Abel, T. 2000, ApJ, 533, 594

Ciardi, B., et al. 2006, Mon. Not. Roy. Astron. Soc., 366, 689
Cohn, J. D., \& Chang, T.-C. 2006, MNRAS, submitted, preprint astro$\mathrm{ph} / 0603438$

Dijkstra, M., Haiman, Z., Rees, M. J., \& Weinberg, D. H. 2004, Astrophys. J., 601, 666

Eisenstein, D. J., \& Hu, W. 1999, ApJ, 511, 5

Fan, X.-H., Carilli, C. L., \& Keating, B. 2006, ARA\&A, in press, preprint astro-ph/0602375 

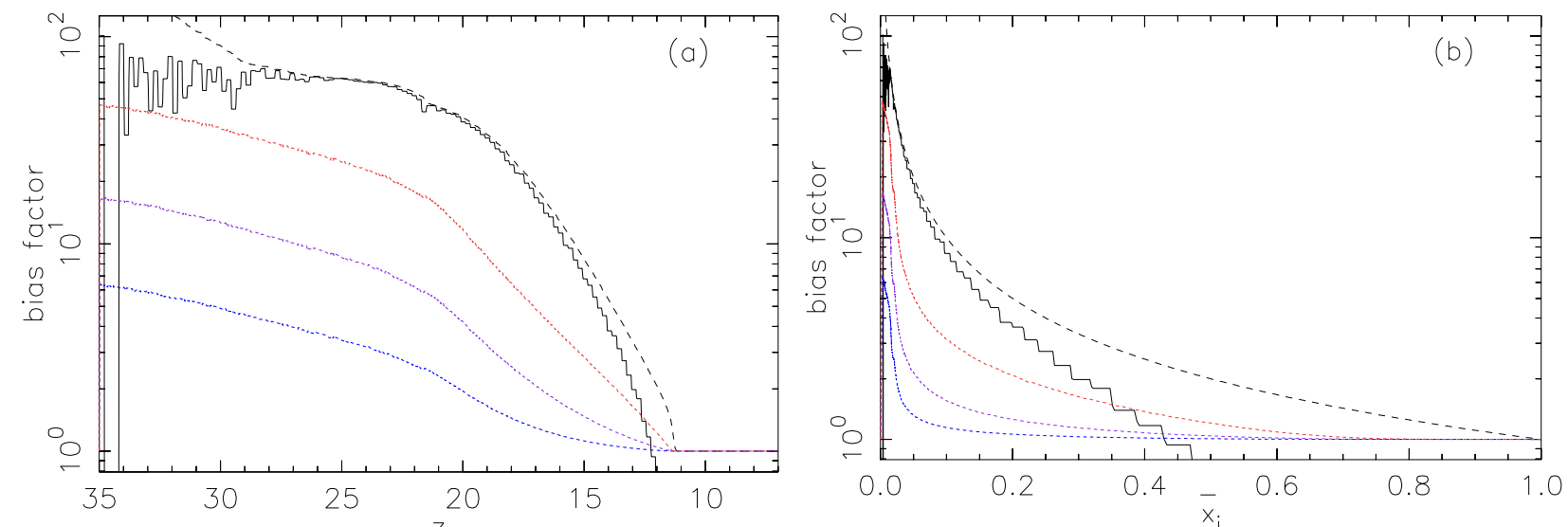

FIG. 6. - Mean bias enhancement factor versus redshift (a) and ionized fraction (b), calculated using equation 15 (top solid curve). Also plotted are the enhancement in the number of neighbors for (lower three curves, top to bottom) large halos, large with minihalos, and minihalos, from equation 16. The long-dashed curve shows $\bar{x}_{\mathrm{i}}^{-1}$ and corresponds to the limiting case when all minihalos are suppressed.

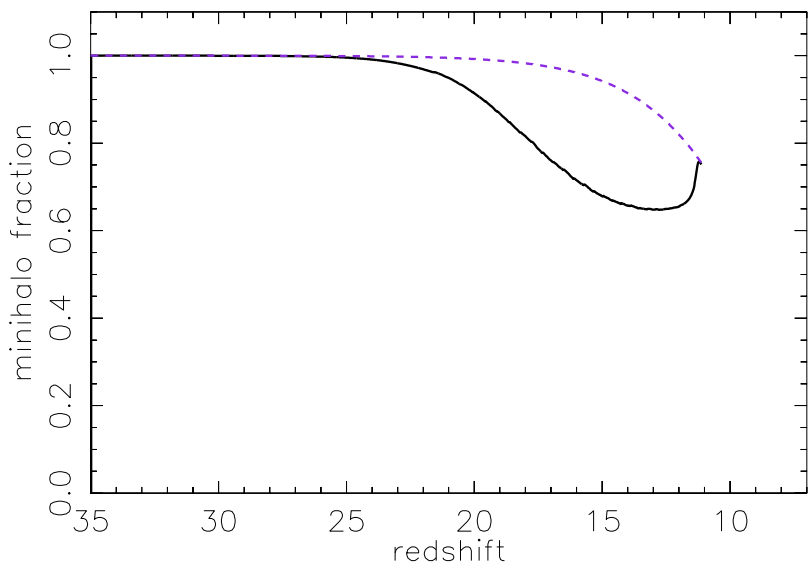

FIG. 7.- Fraction of reionization due to minihalos for the unbiased (dashed) and biased (solid) feedback scenarios.

Furlanetto, S., Zaldarriaga, M., \& Hernquist, L. 2004, Astrophys. J., 613, 1 Furlanetto, S. R., McQuinn, M., \& Hernquist, L. 2006, MNRAS, 365, 115 Furlanetto, S. R., \& Oh, S. P. 2005, Mon. Not. Roy. Astron. Soc., 363, 1031 Haiman, Z., Abel, T., \& Madau, P. 2001, ApJ, 551, 599

Haiman, Z., Abel, T., \& Rees, M. J. 2000, Astrophys. J., 534, 11

Haiman, Z., \& Bryan, G. L. 2006, ApJ, submitted, preprint astro-ph/0603541 Haiman, Z., \& Holder, G. P. 2003, ApJ, 595, 1
Haiman, Z., Rees, M. J., \& Loeb, A. 1997a, ApJ, 476, 458

-. 1997b, ApJ, 484, 985

Iliev, I. T., Scannapieco, E., Martel, H., \& Shapiro, P. R. 2003, MNRAS, 341, 81

Iliev, I. T., Scannapieco, E., \& Shapiro, P. R. 2005, Astrophys. J., 624, 491

Iliev, I. T., et al. 2006, MNRAS, submitted, preprint astro-ph/0603199

Madau, P., Ferrara, A., \& Rees, M. J. 2001, ApJ, 555, 92

Mesinger, A., Bryan, G. L., \& Haiman, Z. 2006, ApJ, submitted

Mesinger, A., \& Haiman, Z. 2004, ApJ, 611, L69

Mo, H. J., \& White, S. D. M. 1996, Mon. Not. Roy. Astron. Soc., 282, 347

Oh, S. P., \& Haiman, Z. 2002, ApJ, 569, 558

Oh, S. P., \& Haiman, Z. 2003, Mon. Not. Roy. Astron. Soc., 346, 456

O'Shea, B. W., Abel, T., Whalen, D., \& Norman, M. L. 2005, ApJ, 628, L5

Ricotti, M., Gnedin, N. Y., \& Shull, J. M. 2002a, ApJ, 575, 33

-. 2002b, ApJ, 575, 49

Scannapieco, E., \& Barkana, R. 2002, ApJ, 571, 585

Scannapieco, E., \& Thacker, R. J. 2005, Astrophys. J., 619, 1

Schaerer, D. 2002, A\&A, 382, 28

Sheth, R. K., \& Tormen, G. 1999, Mon. Not. Roy. Astron. Soc., 308, 119

Spergel, D. N., Verde, L., Peiris, H. V., Komatsu, E., Nolta, M. R., Bennett, C. L., Halpern, M., Hinshaw, G., Jarosik, N., Kogut, A., Limon, M., Meyer, S. S., Page, L., Tucker, G. S., Weiland, J. L., Wollack, E., \& Wright, E. L. 2003, Astrophys. J. Suppl., 148, 175

Spergel, D. N., et al. 2006, ApJ, submitted, preprint astro-ph/0603449

Thoul, A. A., \& Weinberg, D. H. 1996, ApJ, 465, 608

Tumlinson, J., \& Shull, J. M. 2000, ApJ, 528, L65

Wyithe, J. S. B., \& Loeb, A. 2003, ApJ, 588, L69

Zhang, J., \& Hui, L. 2005, ApJ, submitted, preprint astro-ph/0508384 